\title{
HÁBITOS DE ALIMENTACIÓN Y ACTIVIDAD FÍSICA EN ESTUDIANTES UNIVERSITARIOS
}

\section{EATING HABITS AND PHYSICAL ACTIVITY IN UNIVERSITY STUDENTS}

\author{
Luis Espinoza O., Fernando Rodríguez R., \\ Jorge Gálvez C., Norman MacMillan K. \\ Escuela de Educación Física, Facultad de Filosofía y Educación, \\ Pontificia Universidad Católica de Valparaíso, Valparaíso, Chile.
}

\begin{abstract}
During university life students should consolidate healthy habits, presumably acquired during formal education, which should eventually be reflected in the professional adult life. This study aims to determine the influence of the universities on eating habits and physical activity of students. Food habits and physical activity were evaluated in a convenience sample of 169 students of the Pontificia Universidad Católica de Valparaíso attending different courses. The students in this study had been attending the fundamental studies "Autocuidado y Vida Saludable" (Self-care and Healthy Life) given in 2010. Two surveys were taken: Quality of the Food Service (CASEDA) and Quality of the Service of University Sports (CASEDU). They also completed basic anthropometric measurements, blood pressure tests, resting heart rate and blood glucose and triglycerides. The results showed that students have a poor perception of the quality of food service and although they indicated that there is a wide range of healthy and unhealthy foods; they tend to choose foods rich in carbohydrates and fats. This situation adds to their lack of physical activity data. It also shows that in women predominated high levels of triglyceride levels (161mg/dl) and in the male group predominated high blood pressure $(132 \mathrm{mmHg})$. These facts underscore the need to take action at the university to enable students to acquire proper eating habits and provide a range of physical activities which will allow students to better use of their free time at college.
\end{abstract}

Key words: Nutrition, physical activity, university students.

Este trabajo fue recibido el 28 de Abril de 2011 y aceptado para ser publicado el 6 de Julio de 2011.

\section{INTRODUCCIÓN}

Los hábitos de vida saludable en nuestra población, provocan una serie de consecuencias que perjudican el estado de salud de las personas y finalmente influyen en su calidad de vida.

Llevar un estilo de vida saludable, está relacionado con la disminución de la enfermedades crónicas no trasmisibles $(1,2)$. Una etapa crítica en la adquisición de estos buenos hábitos, ocurre en la infancia (3), pero también en la vida universitaria (4), donde los estudiantes pasan de una rutina regular de actividad física a nivel escolar a una rutina cargada de inactividad física (5), típica de la vida universitaria (6). Finalmente se refleja en hábitos de vida poco saludables en la vida adulta profesional (7).

Algunos estudiantes que provienen de lugares lejanos a los centros universitarios de estudios, empeoran su estilo de alimentación, producto de la falta de tiempo (4), para cocinar, comer o conocer la preparación de alimentos saludables que probablemente en sus casas acostumbraban a consumir en su etapa escolar.

Ambos factores influyen fuertemente en el aumento de las posibilidades de tener sobrepeso, grasa corporal aumentada y otros factores de riesgo de la enfermedad cardiovascular (8).

El siguiente estudio pretende determinar la influencia de los centros universitarios, en los hábitos de alimentación saludable y de actividad física de los estudiantes. 


\section{SUJETOS Y MÉTODOS}

De los estudiantes de la Pontificia Universidad Católica de Valparaíso (PUCV), se tomó a la totalidad de los 169 estudiantes que asistieron a la asignatura de estudios fundamentales "Autocuidado y vida saludable", durante el año 2010, quienes procedían de 19 carreras de distintas facultades de la universidad, cuyos años de ingreso eran entre los años 2000 y 2010 y con una edad de entre 18 a 29 años (tabla 1).

Las mediciones, pruebas de laboratorio y encuestas aplicadas fueron informadas a los sujetos, dando sus características y métodos de proceder a través de un "consentimiento informado", accediendo a participar de forma voluntaria en el estudio y con la aprobación del Comité de Etica que regula el proyecto en el que enmarca la investigación.

Se aplicaron dos encuestas: Calidad del Servicio de Alimentación (CASEDA) y Calidad de Servicio de Deporte Universitario (CASEDU); además se realizó una medición antropométrica, medición de presión arterial, frecuencia cardíaca y pruebas de campo para la determinación de la glicemia y triglicéridos sanguíneos.

La encuesta CASEDA considera datos básicos del encuestado tales como género, año de ingreso, edad y carrera. Es un instrumento validado en un proyecto de Investigación, aprobado y financiado por la Dirección de Investigación e Innovación (DII) de la Pontificia Universidad Católica de Valparaíso. Se pregunta a los estudiantes si su facultad cuenta con un lugar donde adquirir alimentos, si solo dispone de bocadillos o también comida caliente preparada. Entregando un listado de distintos tipos de alimentos, se solicita al alumno señalar cuales están disponibles para adquirir en los casinos, kioscos y cafeterías. Además se solicita ordenar por preferencia de consumo ciertos alimentos y evaluar la calidad del servicio de alimentación de la universidad y la disponibilidad de alimentos con una calificación de 1 a 7 , donde 1 es lo más deficiente y 7 es excelente.
La encuesta CASEDU también es un instrumento validado en un proyecto de Investigación, aprobado y financiado por la DII de la Pontificia Universidad Católica de Valparaíso. Se pregunta a los alumnos si realizan actividad física, cuales son sus motivos, cuáles son sus preferencias para realizara actividad física en la universidad, en qué lugar la realizan, si asisten a un programa regular y si tiene asesoría profesional. Además se les solicita calificar de 1 a 7 , donde 1 es lo más deficiente y 7 es excelente, la calidad del servicio profesional y la oferta de actividad física entregada por la universidad,

También se realizaron evaluaciones antropométricas para la descripción de la composición corporal, utilizando las formulas de Yuhasz (9) y modificado por Faulkner quién mejora la fórmula en un grupo de nadadores (10).

La fórmula utilizada para la estimación de la masa grasa es, $\%$ Peso Graso $=0,153 *($ Suma de 6 Pliegues $)+$ 5,783. Los pliegues medidos son tricipital, subescapular, supracrestideo, abdominal, muslo frontal y pierna, utilizando un plicómetro plástico Slimguide. Se considera el IMC el que se obtiene dividiendo el peso (en $\mathrm{kg}$ ) por la estatura (en metros) elevado al cuadrado. El peso y la estatura se evalúan en una balanza Seca 700 con estadiómetro incorporado. Estas medidas se realizan de pié con la menor ropa posible y después del vaciado urinario. La estatura se registra con el sujeto ubicado en el plano de Frankfort para evitar errores en la medición.

La presión arterial se medió con un sistema oscilométrico BRAUN BP4010. Este procedimiento se realiza con el alumno sentado y después de 5 min de reposo. El dispositivo se ubica en el brazo izquierdo y se repite el procedimiento tres veces para dejar como resultado final, el promedio de las tres mediciones. La frecuencia cardíaca se mide con un monitor cardíaco Polar S1 con una cinta ubicada a nivel esternal y un registro en el reloj de pulsera. La valoración de la glicemia se determinó con un dispositivo portátil Accu Chek Active

\section{TABLA 1}

Características de los sujetos evaluados, según el sexo (n=169).

FEMENINO $(n=94)$

$\mathrm{X} \pm \mathrm{DE}$

EDAD (años)

PESO (kg)

TALLA (cm)
$22,4 \pm 1,9$

$58,2 \pm 7,3$

$159,6 \pm 6,0$
MASCULINO $(n=75)$

$\mathrm{X} \pm \mathrm{DE}$

$23,4 \pm 2,0$

$73,5 \pm 10,8$

$173,7 \pm 5,2$

$\mathrm{X}=$ promedio $; \mathrm{DE}=$ desviación estándar. 
con cintas reactivas que reaccionan a la luz infrarroja. Finalmente los triglicéridos sanguíneos se determinaron con un dispositivo ACCUTREND PLUS. No se evaluó el colesterol total, porque el dispositivo disponible en este estudio no discriminaba entre HDL y LDL, por lo que ese valor no hubiese sido un buen indicador para nuestros objetivos.

Todas las evaluaciones se realizaron a cada alumno en una sola mañana, en ayunas y después del vaciado urinario.

Los resultados se registraron en planillas de Microsoft Excel de Office 2007. Los resultados se analizaron en la plataforma SPSS versión $n^{\circ} 17$ para la obtención de la significancia en una prueba T-Student considerando un nivel significativo cuando $\mathrm{p}<0,05$. En este procedimiento se compararon las variables por sexo, considerando la misma varianza y con valores no pareados, esto por las diferencias en el " $n$ " entre los sujetos de los grupos masculino y femenino.

\section{RESULTADOS \\ Encuesta CASEDA}

De la encuesta CASEDA se toman los resultados que se consideran más relevantes de las 9 preguntas que constaba este instrumento. Sobre el servicio de alimentación de las diferentes facultades de la Pontificia Universidad Católica de Valparaíso. Ante la pregunta: en el centro universitario donde estudiar, ¿cuenta con servicio de alimentación, casino, cafetería o kiosco? El 99,3\% de los encuestados afirmó contar con alguno de estos servicios. Además, el 78,5\% de los estudiantes manifiesta contar con servicio de menú de comida preparada, que consta de al menos un plato principal y ensalada.

Al consultarle a los estudiantes sobre la disponibilidad de ciertos alimentos, los tres primeros altos porcentajes de presencia en los kioscos o casinos son, 90,5\% señala que en dicho servicio de alimentación se expenden lácteos, el 80,4\% señala que se venden completos (hot dog), un 79,7\% señala que expenden galletas de avena. En último lugar, $18,9 \%$ de los estudiantes señala que existen a la venta frutos secos (figura 1).

Otra pregunta de la encuesta CASEDA, se refiere a la evaluación del servicio de alimentación prestado por la universidad, donde se valora con una nota 1 como lo más negativo y 7 como la calificación máxima, respecto a la disponibilidad y oferta de alimentos, así como a la comodidad y horario del servicio de alimentación (tabla 2).

La figura 2 ilustra las preferencias de consumo de los estudiantes en los centros de alimentación de la universidad, ante la pregunta ¿qué tipo de alimento es el que comúnmente compra y consume en los centros de alimentación de la universidad? la primera elección de los alumnos son las golosinas, seguida por los lácteos, comida rápida, bebidas y finalmente productos con fibra.

Esta misma pregunta se analizó por sexo; la figura 3 muestra que $26,3 \%$ de las mujeres prefieren las golosinas

\section{FIGURA 1}

Disponibilidad de alimentos saludables y no saludables en los distintos servicios de alimentación de las facultades, según la pregunta: Tu centro de alimentos, ¿expende algunos de estos alimentos?

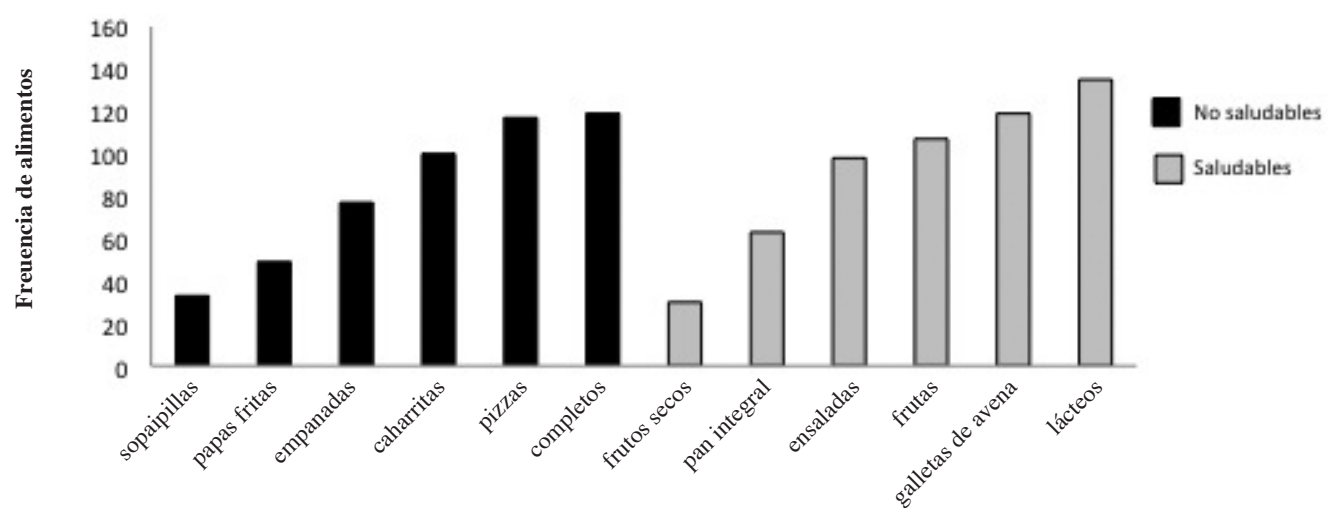

Alimentos identificados con mayor disponibilidad 
como primera opción, en tanto los hombres prefieren la comida rápida en primera opción con un $31,3 \%$. El producto menos preferido por las mujeres es la comida rápida $(12,6 \%)$ y para los hombres los productos con fibra $(12,5 \%)$.

\section{Encuesta CADESU}

De las 10 preguntas que contenía el instrumento CASEDU, se presentan los resultados más relevantes.

A la pregunta $¿$ Te agrada hacer ejercicio?, 97,3\% del grupo responde que si, en cambio $87,2 \%$ del grupo de mujeres, responde afirmativamente.

Los estudiantes que no realizan actividad física, señalan que no tienen tiempo o no se hacen el tiempo $(35 \%)$, en segundo lugar porque no están dadas las condiciones para la práctica $(24 \%)$, otras causas institucionales
(16\%) y otras causas personales $(15 \%)$.

Respecto de la pregunta ¿Realiza ejercicio en algún programa de actividad física de la Universidad?, 42,7\% del grupo masculino y el $29,8 \%$ del grupo femenino responden que si.

De los alumnos que si realizan en la universidad, el $42 \%$ lo realiza con objetivos de salud, $37 \%$ con objetivos recreativos, $16 \%$ con motivaciones deportivas y $5 \%$ por otras razones.

De acuerdo a la pregunta ¿Cuáles son las causas para realizar ejercicio fuera de la Universidad?, el grupo completo de estudiantes, señala como razón principal, el que no existan las condiciones adecuadas para práctica de ejercicio (37\%), como espacios físicos, implementación entre otras. En segundo lugar que no hay ofertas de actividades que son de preferencia de los alumnos

\section{TABLA 2}

Evaluación del servicio de la alimentación de la universidad, respecto de la disponibilidad y oferta de los alimentos y a la comodidad y horario de atención de los centros de alimentación.

Disponibilidad y oferta

$$
\mathrm{X} \pm \mathrm{DE}
$$

$$
4,1 \pm 1,4
$$

Comodidad y horario de atención $\mathrm{X} \pm \mathrm{DE}$

$4,8 \pm 1,4$

$\mathrm{X}=$ promedio; $\mathrm{DE}=$ desviación estándar en una escala de 1 a 7 donde 1 es deficiente y 7 es excelente.

\section{FIGURA 2}

Consumo de alimentos según la preferencia de los alumnos en los distintos campus y facultades.

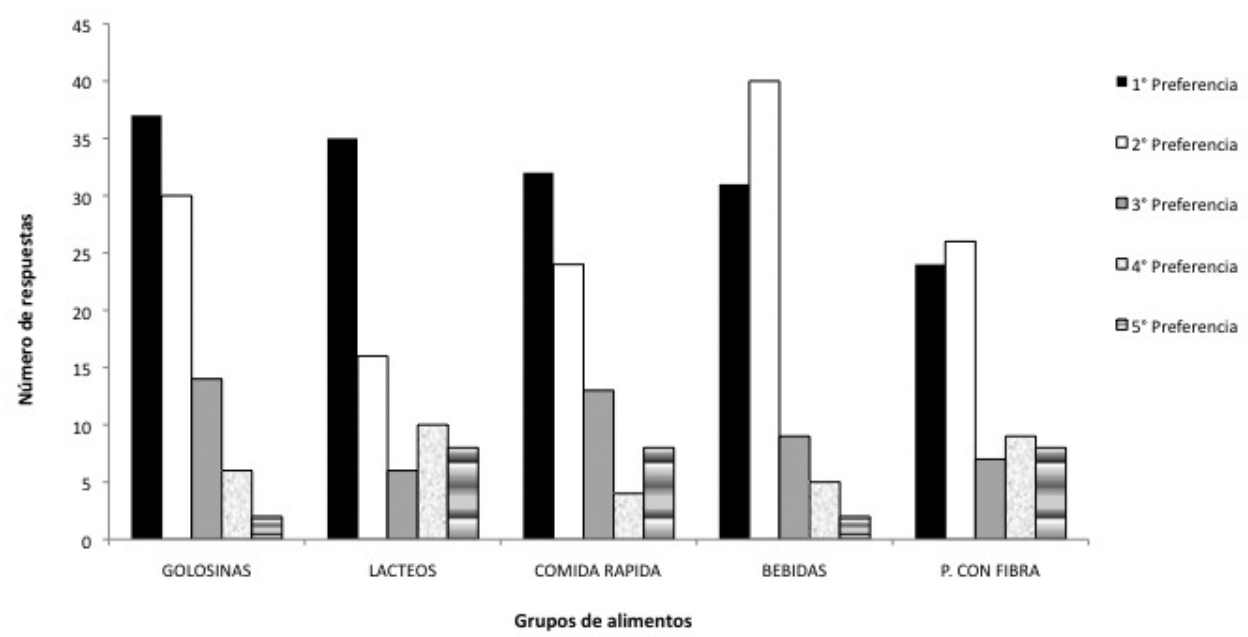


(20\%). La tercera razón más recurrente es que no hay cupos suficientes para las actividades ofrecidas en la Universidad. Del grupo, 27\% responde por otras causas, como que le incomoda llevar ropa de cambio a la universidad, que los horarios no les son cómodos, que no hay donde ducharse, que no tienen dinero para comprar los implementos deportivos personales, entre otras.

\section{Antropometría y otras variables}

En la tabla 3 se aprecian los resultados de la antro-

\section{FIGURA 3}

\section{Preferencias de consumo de alimentos, según sexo.}

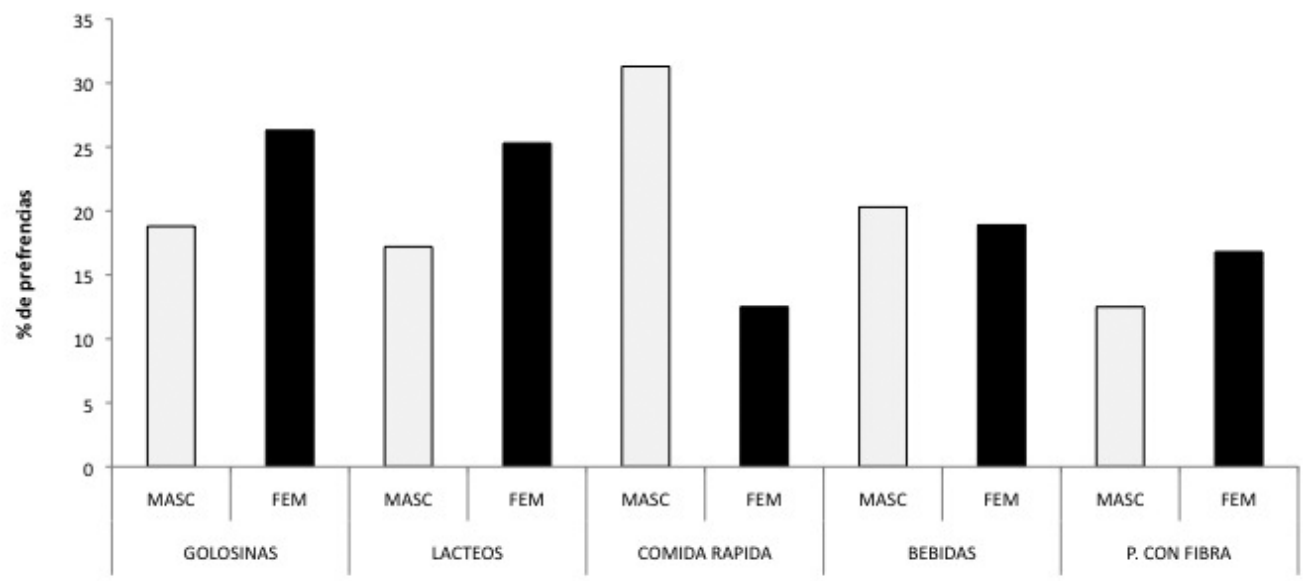

TABLA 3

Variables antropométricas.

\begin{tabular}{lcc} 
& FEMENINO & MASCULINO \\
& $\mathbf{X} \pm \mathbf{D E}$ & $\mathbf{X} \pm \mathbf{D E}$ \\
\hline ICC $(*)$ & $0,7 \pm 0,04$ & $0,8 \pm 0,1$ \\
IMC $(*)$ & $22,9 \pm 3,0$ & $24,3 \pm 3,1$ \\
Bajo peso $(\%)$ & $15,0-$ & $3,0-$ \\
Normopeso (\%) & $69,0-$ & $60,0-$ \\
Sobrepeso (\%) & $14,0-$ & $32,0-$ \\
Obesidad (\%) & $2,0-$ & $5,0-$ \\
SUM PL $(*)$ & $81,2 \pm 22,4$ & $71,4 \pm 22,7$ \\
\% MG $(*)$ & $19,6 \pm 4,9$ & $16,7 \pm 3,5$ \\
\hline (*) Diferencias significativas p<0,05 entre sexos em uma prueba t-student. & \\
X= promedio; DE= desviación estándar. & \\
Normalidad (WHO): & & \\
ICC; $0,71-0,85$ Femenino y $0,78-0,94$ Masculino. & & \\
IMC; $18,5-24,9$. & & \\
$\%$ Grasa; 20 y 25 femenino y 10-15 Masculino. &
\end{tabular}


pometría básica para Índice Cintura Cadera (ICC), Índice de Masa Corporal (IMC), sumatoria de 6 pliegues y el porcentaje de masa grasa (\% MG). Los valores de ICC e IMC se encuentran dentro de los rangos normales para ambos sexos, no así la sumatoria de 6 pliegues ni el \% MG, los cuales son elevados.

La tabla 4 muestra las variables glicemia (GL) y presión sistólica (PS), siendo significativamente mayores en el grupo femenino que en el grupo masculino.

Los triglicéridos (TG) y la frecuencia cardíaca $(\mathrm{FC})$, son mayores en el grupo de mujeres.

\section{DISCUSIÓN}

Los estudiantes evaluados señalan contar con un servicio de alimentación que en general cumple los requerimientos de horario, comodidad y disponibilidad de alimentos variados, pero al momento de calificar el servicio, la nota alcanza a ser apenas suficiente (tabla 2), por lo que tienen una percepción de la calidad del servicio de alimentación baja.

Señalan también que existe una amplia oferta de alimentos que podemos clasificar como saludables y no saludables (figura 2). Pese a esto los estudiantes elijen mal los alimentos, adquiriendo principalmente alimentos clasificados como poco saludables. En esta elección es independiente del costo del producto elegido, ya que por ejemplo comprar frutas o lácteos son de un valor económico más bajo que las bebidas gaseosas o comidas preparadas no saludables como hog dog, empanadas o emparedado.

Es necesario destacar que la "comida rápida" es la primera preferencia del grupo masculino para adquirir alimentos en la universidad y las "golosinas" la primera preferencia para el grupo femenino (figura 3). Los productos con fibra son la última opción de compra de alimentos. Resultados similares se obtuvieron un estudio realizado en estudiantes universitarios del Campus Antumapu de la Universidad de Chile (11), donde el consumo calórico total, la ingesta de carbohidratos y lípidos era significativamente más alto en los estudiantes de sexo masculino que en los de sexo femenino y ninguno de los estudiantes alcanzaba la recomendación diaria de fibra dietética.

Esta condición se suma a la falta de práctica de actividad física de los estudiantes, quienes señalan hacer ejercicio preferentemente fuera de la universidad. Las razones son diversas, pero señalan que la universidad no cuenta con los espacios ni las ofertas de programas que los alumnos necesitan.

En consecuencia los alumnos presentan características de IMC promedio en el límite del sobrepeso, presión sanguínea promedio elevada en el grupo masculino y triglicéridos promedio elevados en el grupo femenino.

Otro antecedente importante es el referido a los marcadores fisiológicos valorados, donde la presión arterial (PA) en el grupo masculino se encuentra en una categoría normal alta según la OMS/Sociedad Internacional de Hipertensión y Joint National Committee on Prevention for High Blood Pressure (12).

Esta condición de PA alterada provoca una serie de efectos perjudiciales a la salud cardiovascular (13) que se puede ver aumentado con estados de estrés típicos de

\section{TABLA 4}

Variables fisiológicas diferenciadas por género.

\begin{tabular}{lcc} 
& $\begin{array}{c}\text { FEMENINO } \\
\text { X + DE }\end{array}$ & $\begin{array}{c}\text { MASCULINO } \\
\text { X + DE }\end{array}$ \\
\hline GL $\left(^{*}\right)$ & $81,0+8,3$ & $87,5+8,4$ \\
TG $\left(^{*}\right)$ & $161,7+90,4$ & $112,9+50,9$ \\
PS $\left(^{*}\right)$ & $120,2+16,4$ & $132,1+19,6$ \\
PD & $87,4+15,5$ & $87,2+15,1$ \\
FC $(*)$ & $81,6+11,8$ & $72,2+18,8$ \\
\hline (*) Diferencias significativas P<0,05 entre sexos en una prueba t-student. & \\
X= promedio; DE= desviación estándar. & & \\
Normalidad $($ Clínica Santa María, Chile): & & \\
GL; $70-100 \mathrm{mg} / \mathrm{dL}$ & & \\
TG; $<150 \mathrm{mg} / \mathrm{dL}$ & & \\
PS; $<139 \mathrm{~mm} / \mathrm{Hg}$ & & \\
PD; $<89 \mathrm{~mm} / \mathrm{Hg}$ & &
\end{tabular}


poblaciones universitarias.

En un estudio hecho en universitarios en la Universidad de Talca (14), se presentan valores de PS y PD más bajas que este grupo de estudio, siendo los valores de este estudio, preocupantes para el estado de salud de los estudiantes. Esta condición puede ser empeorada por estados de estrés a los que son sometidos los estudiantes, aumentando las posibilidades de padecer hipertensión arterial en este período etario.

Los TG séricos se encuentran en valores elevados en el grupo de mujeres, ya que los valores obtenidos están clasificados como en el límite alto (15) siendo esto, una representación de dieta inadecuada y de la clara falta de ejercicio de estas estudiantes.

Otro elemento a considerar es la motivación para la práctica de actividad física, donde los estudiantes manifiestan, principalmente no tener tiempo, similar a lo encontrado en un estudio de Olivares et al. donde el 64\% de los estudiantes señala esta como la principal razón (16).

La guía para universidades saludables ha sido un referente para tomar acciones que encaminen los cambios conductuales en la salud de los estudiantes y que señala la importancia de promover los hábitos de vida saludable en estudiantes universitarios que egresan, es una buena manera de fomentar estos estilos de vida en las instituciones en las que trabajan (17).

Estos antecedentes ponen de manifiesto la necesidad de que las universidades tomen parte responsable en las acciones que permitan a los alumnos aprender de hábitos alimenticios adecuados (18), y que puedan elegir bien los alimentos a adquirir, además de entregar una oferta amplia de actividades físicas y de oportunidades de espacios (16) que permitan a los estudiantes ocupar sus tiempos libres, dentro de la universidad para la práctica de actividad física regular y disminuir con esto los factores de riesgo a la salud que se presentan en esta etapa universitaria y evitar su empeoramiento en la vida adulta fuera de la universidad.

\section{RESUMEN}

Durante la vida universitaria debieran consolidarse los hábitos saludables, supuestamente adquiridos durante la enseñanza escolar, los que finalmente deberían verse reflejados en el profesional adulto. En este estudio se pretende determinar la influencia de los centros universitarios, en los hábitos de alimentación y actividad física de los estudiantes. Se valúan los hábitos alimentarios y de actividad física de una muestra intencionada de 169 estudiantes de diferentes carreras de la Pontificia Universidad Católica de Valparaíso, que asisten a la asignatura de estudios fundamentales
"Autocuidado y vida saludable", dictada durante el año 2010. Se aplicaron dos encuestas: Calidad del Servicio de Alimentación (CASEDA) y Calidad de Servicio de Deporte Universitario (CASEDU). Además se realizaron antropométrica básica, determinación de la presión arterial, frecuencia cardíaca de reposo y pruebas sanguíneas para la determinación de glicemia y trigliceridemia. Los resultados arrojaron que los estudiantes tienen una mala percepción de la calidad del servicio de alimentación y aunque señalan que existe una amplia oferta de alimentos saludables y no saludables, tienden a elegir alimentos ricos en carbohidratos y lípidos. Esta situación se suma a su escaza práctica de actividad física. Se aprecia además que en el grupo femenino predominan niveles de triglicéridos elevados (161,7 mg/dL) y en el grupo masculino, hipertensión arterial elevada $(132,1 \mathrm{mmHg})$. Estos antecedentes ponen de manifiesto la necesidad tomar acciones a nivel universitario que permitan a los alumnos adquirir hábitos alimentarios adecuados y entregar una oferta de actividades físicas que permitan a los estudiantes ocupar sus tiempos libres dentro de la universidad.

Palabras clave: Nutrición, actividad física, estudiantes universitarios.

Dirigir la correspondencia a:

\section{Profesor}

Luis Espinoza Oteiza

Pontificia Universidad Católica de Valparaíso

Avda. El Bosque 1290, Santa Inés,

Viña del Mar, Chile

Fono: 322274381

e-mail: lespinoz@ucv.cl

Agradecimientos: Agradecemos a la Dirección de Investigación e Innovación (DII), por su constante esfuerzo y apoyo a las iniciativas a proyectos internos en la Pontificia Universidad católica de Valparaíso; especialmente a nuestros alumnos de Autocuidado y Vida Saludable, que semestre a semestre nos motivan a seguir trabajando.

\section{BIBLIOGRAFÍA}

1. Vio F, Albala C, Crovetto M. Promoción de salud en la transición epidemiológica de Chile. Rev Chil Nutr 2000; 27(1): 21-9.

2. Vio F, Albala C. Epidemiología de la obesidad en Chile. Rev Chil Nutr 2000; 27: 97-112.

3. Busdiecker S., Castillo C., Salas I. Cambios en los hábitos de alimentación durante la infancia: una visión antropológica. Rev Chil Pediatr 2000; 71 (1): 5-11. 
4. Troncoso P Claudia, Amaya P Juan Pablo. Factores sociales en las conductas alimentarias de estudiantes universitarios. Rev Chil Nutr 2009; 36(4): 1090-1097.

5. Aránguiz A, Hugo et al. Estudio descriptivo, comparativo y correlacional del estado nutricional y condición cardiorrespiratoria en estudiantes universitarios de Chile. Rev Chil Nutr 2010; 37 (1): 70-8.

6. MacMillan K Norman. Valoración de hábitos de alimentación, actividad física y condición nutricional en estudiantes de la Pontificia Universidad Católica de Valparaíso. Rev Chil Nutr 2007: 34(4): 330-6.

7. López E Miguel Ángel. Etapas del cambio conductual ante la ingesta de frutas y verduras, control de peso y ejercicio físico de estudiantes de la Universidad del Desarrollo, sede Concepción, Chile. Rev Chil Nutr 2008; 35(3): 215-24.

8. Whitlock G, Lewington S, Sherliker P, et al. Bodymass index and cause-specific mortality in 900000 adults: collaborative analyses of 57 prospective studies. Lancet 2009; 28;373(9669):1083-96.

9. Yuhasz MS. Physical fitness manual. London, Ontario: University of Western Ontario. 1974.

10. Faulkner JA. Physiology of swimming and diving. En: Falls H. Exercise physiology. Baltimore. Academic Press. 1968.

11. Durán S, Castillo M, Vio F. Diferencias en la calidad de vida de estudiantes universitarios de diferente año de ingreso del campus Antumapu. Rev Chil Nutr 2009; 36(3):200-9.
12. 1999 World Health Organization-International Society of Hypertension Guidelines for the Management of Hypertension. Gudelines Subcommittee. J Hypertens 1999;17:151-83.

13. Persu A, De Plaen JF. Recent insights in the development of organ damage caused by hypertension. Acta Cardiol 2004;59:369-81.

14. Palomo I, Torres G, Alarcón M, Maragaño P, Leiva $\mathrm{E}$, Mujica V. Alta prevalencia de factores de riesgo cardiovascular clásicos, en una población de estudiantes universitarios de la región centro-sur de Chile. Rev Esp Cardiol 2006; 59: 1099-105.

15. Grundy SM, Cleeman JI, Merz CN, Brewer HB Jr, Clark LT, Hunninghake DB, et al. Implications of recent clinical trials for the National Cholesterol Education Program Adult Treatment Panel III guidelines. Circulation 2004;110:227-39.

16. Olivares C, Sonia; Lera M, Lydia Y Bustos Z, Nelly. Etapas del cambio, beneficios y barreras en actividad física y consumo de frutas y verduras en estudiantes universitarios de Santiago de Chile. Rev Chil Nutr 2008; 35 (1); 25-35.

17. Lange I, Vio F. Guía para universidades saludables y otras instituciones de educación superior Santiago: Vida Chile, OPS/OMS/INTA/PUC; 2006.

18. Mardones H, María Angélica, Olivares C, Sonia, Araneda F, Jacqueline et al. Etapas del cambio relacionadas con el consumo de frutas y verduras, actividad física y control del peso en estudiantes universitarios chilenos. ALAN 2009: 59 (3); 304-9. 